NASZA DERMATOLOGIA Online OUR DERMATOLOGY Online

Source of Support: Nil

Competing Interests: None

\section{SEPTAL PANNICULITIS: CLINICO-PATHOLOGICAL REVIEW OF THE LITERATURE AND CASE PRESENTATION}

\author{
Gabriela Martinez Braga, Beatriz Di Martino Ortiz \\ 'Private Medical Center „La Costa”, Asunción, Paraguay \\ 2Department of Dermatology, Clinical Hospital, Faculty of Medical Sciences, \\ National University of Asunción, Asunción, Paraguay
}

Corresponding author: Ass.Prof. Beatriz Di Martino Ortiz

beatrizdimartino@gmail.com

\begin{abstract}
The panniculitides comprise a large and heterogeneous group of diseases, with a high prevalence in the general population, in which the inflammatory process affects the subcutaneous tissue. We briefly review the classification, clinical and histology of the primary panniculitides and present a representative case.
\end{abstract}

Key words: panniculitis; septal panniculitis; lobular panniculitis; erythema nodosum

\section{Introduction}

The panniculitides comprise a large and heterogeneous group of diseases, with a high prevalence in the general population, in which the inflammatory process affects the subcutaneous tissue $[1,2]$. We briefly review the classification, clinical and histology of the primary panniculitides and present a representative case. The hypodermis or subcutaneous tissue forms the deepest part of the skin, embryologically derived from the mesenchym whose cells give rise to adipocytes. The basic building block of the hypodermis is the fat cell or adipocyte; these represent a special line of connective tissue cells. They are organized into lobules of about one centimeter in diameter.

The lobules are separated and supported by connective tissue through which pass the blood, lymph vessels and nerves [3]. The subcutaneous tissue of the body acts as insulation, energy reservoir, cushion and protector of the skin and allows mobility over adjacent structures [4].

The nutrient artery supplyies the center of the lobe with drainage into venules located in fibrous septa.

Consequently, interference with the arterial supply produces changes in the lobe (lobular panniculitis), while venous disorders are manifested by disturbances in the paraseptal regions (septal panniculitis). Inflammation of small veins will result in a septal panniculitis with localization of the inflammatory process in the lower dermis. Involvement of the arterial blood supply, for example, in vasculitis, produces lobular panniculitis. Other mechanisms are involved in some of the diseases that result in a lobular panniculitis.

From a clinical point of view, panniculitis manifests as more or less well-defined erythematous violaceous nodules or lumps, which coalesce to form indurated erythematous plaques that may be painful.

They are often seen in the lower extremities, but may affect any site where fat tissue is more abundant, as in the mammary regions.

Based on the clinical features, location, inicial lesiones and morphology of injuries, we can perform a diagnostic approach. However to make a firm diagnosis a skin biopsy including subcutaneous tissue should be performed.

\section{Classification}

Panniculitis classification is performed in accordance with histological examination, the location of the inflammatory process, involvement or not of the blood vessels and the predominant cell type in the infiltrate.

If the inflammatory process affects mainly the fibrous trabeculae or septa the panniculitis is called septal, and lobular or lobar when lobes are predominantly affected.

The distinction between septal and lobular panniculitis is usually artificial, since there are no purely septal or lobular panniculitides. 
It is considered that all panniculitides are mixed because they all have an inflammatory infiltrate able to affect the septum and lobes, but the inflammatory infiltrate is more abundant in one or other of these two components, so the diagnosis should be noted mainly as septal or mostly lobular.

The presence or absence of vasculitis divides panniculitis into four main groups: 1. lobular panniculitis without vasculitis, 2. lobular panniculitis with vasculitis, 3 . septal panniculitis without vasculitis and 4. septal panniculitis with vasculitis (Tabl. I) .

\section{Septal panniculitis without vasculitis}

Erythema Nodosum

Erythema nodosum was described by Robert Willan in 1798, later by Wilson in 1842, and Strand in 1866. It is the most common form of panniculitis [3].

Erythema nodosum occurs in about 1-5 per 100,000 inhabitants. In adults it is more common in women with a ratio of 1:6 to men. Before puberty the incidence is similar in both sexes and decreases after menopause in women $[3,5]$.

\begin{tabular}{|c|}
\hline Predominantly septal panniculitis \\
\hline $\begin{array}{l}\text { - No vasculitis } \\
\text { Erythema nodosum. } \\
\text { Panniculitis of morphea-scleroderma. } \\
\text { Necrobiosis lipoidica. }\end{array}$ \\
\hline $\begin{array}{l}\text { - With vasculitis * } \\
\text { Cutaneous polyarteritis nodosa. } \\
\text { Surface migratory thrombophlebitis. }\end{array}$ \\
\hline Predominance of lobular panniculitis \\
\hline $\begin{array}{l}\text { - No vasculitis } \\
\text { Enzymatic: } \\
\text { Pancreatic panniculitis } \\
\text { Panniculitis in alpha -1 antitrypsin deficiency } \\
\text { Immunologic: } \\
\text { Panniculitis of lupus erythematosus } \\
\text { Panniculitis in dermatomyositis } \\
\text { Panniculitis in connective tissue disease } \\
\text { With needle-shaped slits in adipocytes: } \\
\text { Sclerema neonatorum } \\
\text { Subcutaneous fat necrosis of the newborn } \\
\text { Post steroid panniculitis } \\
\text { Lipodistrophic: } \\
\text { Lipoatrophy: partial, full, localized, associated with HIV, gynoid (cellulitis), membranous. } \\
\text { Lipohypertrophy } \\
\text { Traumatic/Physical: } \\
\text { Cold Panniculitis } \\
\text { Sclerosing lipogranuloma } \\
\text { Panniculitis by injections } \\
\text { Panniculitis by blunt trauma } \\
\text { Panniculitis factitia, traumatic fat necrosis and encapsulated fat necrosis. } \\
\text { Panniculitis induced by infection. } \\
\text { Lipodermatosclerosis. } \\
\text { Noninfectious neutrophilic and eosinophilic panniculitis. } \\
\text { Malignancies: } \\
\text { Subcutaneous malignant infiltrates. } \\
\text { Histiocytic cytophagic panniculitis. } \\
\text { Subcutaneous panniculitis -like T- cell lymphoma. }\end{array}$ \\
\hline $\begin{array}{l}\text { - With vasculitis } \\
\text { Indurated erythema-nodular vasculitis }\end{array}$ \\
\hline Table I. Panniculitis classification. \\
\hline $\begin{array}{l}\text { * The panniculitis associated with the Involvement of large vessels, polyarteritis nodosa and migratory thrombophlebitis, } \\
\text { usually are located in the vicinity of the vessel Involved. Often have a mixture of septal and lobular features. }\end{array}$ \\
\hline
\end{tabular}


There seems to be no differences between rural and urban. Some studies have shown a higher incidence in winter-spring, summer-autumn, although this appears to be related more to the cause. The most affected age range is between 20 to 30 years [5].

The intimate mechanism or pathogenesis of erythema nodosum is unknown, it is thought that it is an immune-mediated process (multiple immunological mechanisms) or caused by circulating immune cell delayed hypersensitivity reaction (type III and IV of Gell and Coombs) in response to different antigenic stimuli [3].

There is a wide spectrum of etiologic agents that trigger this reaction, although 30 to $50 \%$ of cases are idiopathic. Tuberculosis in adults is the most frequently related entity, followed by reactions to medications, hormonal changes, inflammatory bowel disease and sarcoidosis. In children streptococcal infections and urinary tract infections are the most often associated pathologies (Tabl. II) $[3,4,6]$.

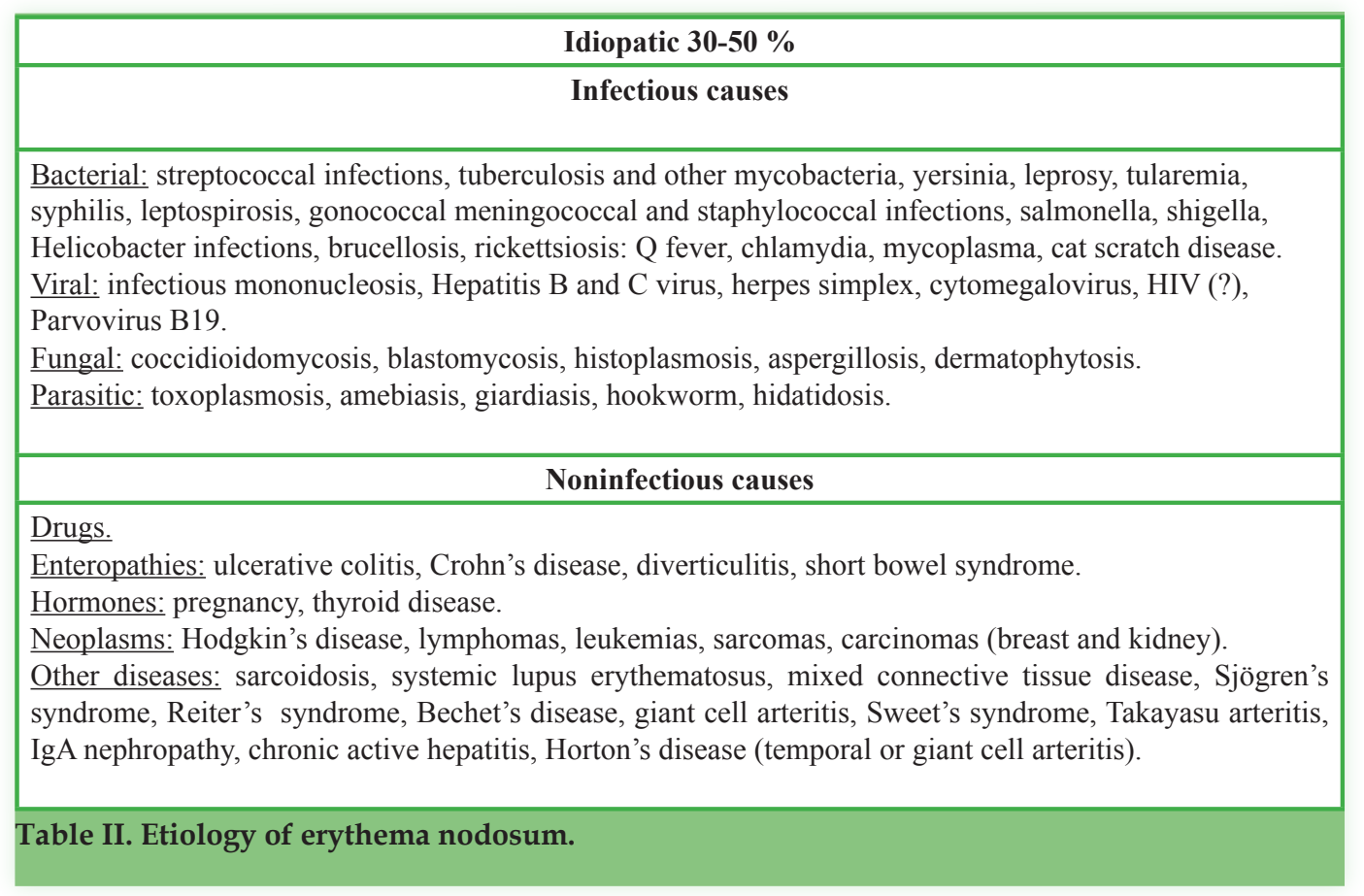

\section{Three clinical forms are distinguished:}

1. Typical: the acute and multi and bilateral lesions form.

2. Migratory: this is usually a single lesion or multiple but grouped asymmetric, less painful and rarely accompanied by systemic symptoms, has a more chronic course without relapsing but growing eccentrically in the middle.

3. Chronic: it is characterized by the existence of single or multiple persistent nodules recurring for months or years in the legs [6].

In the investigations, should be considered as routine tests complete blood cell count with sedimentation rate, platelets, mean cell volume, simple examination of urine sediment, blood chemistry profile with liver, C-reactive protein, ASO and intradermal tests Mantoux - radiography of the chest and abdomen, throat swab, kidney function test. The rest of the scans will be performed according to suspected etiologies $[3,6]$. There is no unanimity among authors about whether the diagnosis should be exclusively clinical or if biopsy is necessary in all cases. The prevailing attitude is to base the diagnosis on clinical grounds when the skin lesions are typical and in the rest, take a biopsy. Etiologic diagnosis is based on clinical and analytical data derived from the suspected pathology. Based on the realization of a good history, physical examination and appropriate laboratory tests.

Biopsy is of a septal panniculitis with small foci of inflammatory cells extending into the adjacent lobular fat. In some cases this overflow of cells is marked and includes foam cells, sometimes associated with focal necrosis of fat cells (not a typical feature). The lobe center is unaffected (allows a distinction from lobular panniculitis). There is also a certain degree of inflammatory cells in the adjacent lower dermis.

In most biopsies the infiltrate is predominantly lymphocytic, but there are a variable number of giant cells of foreign body type and a few eosinophils and histiocytes.

Oval small nodules consisting of histiocytes radially around a central grooved crescent (Miescher radial granulomas) are observed. Tuberculoid well formed granulomas are rare.

The summary of findings by time of evolution is:

- Early/Acute: Neutrophils (can be very abundant in the rare variant of erythema nodosum, suppurativa). Septa broadened by edema and fibrinoid changes.

- Intermediate/Established: mononuclear cells predominate. Miescher granulomas.

- Delayed: Increased fibroblast proliferation with obliteration and fibrosis of the septum. 
Treatment is essentially symptomatic and includes general and anti-inflammatory actions:

1. General measures: bed rest with elevation of the lower extremities, use of compression bandages with elastic stockings. 2. Anti-inflammatory: non steroidal anti-inflammatory drugs (NSAIDs), corticosteroids and potassium iodide:

- NSAIDs. Salicylates are the most commonly used at dose 2-6 $\mathrm{g} /$ day, indomethacin, naproxen and ibuprofen.

- Potassium iodide is orally at doses of 0.5-1 g/day in 3 doses for

2-4 weeks produces rapid symptom response.

- Corticosteroids: systemic corticosteroids should be limited to the most serious cases and those that have not responded to other treatments. Prednisone, 30-60 mg/day is used, in descending dose for $2-3$ weeks in adults and 0.5 to $2 \mathrm{mg} / \mathrm{kg}$ in children. In some recurring or chronic injuries,intralesional corticosteroids have been used.

3. Other treatments: have also been found useful colchicine at doses of $2 \mathrm{mg}$ /day for the first day and then $1 \mathrm{mg}$ /day for 2-3 weeks. It quotes the etanercept and infliximab as possible treatments in cases of chronic erythema nodosum $[6,7,9]$. The main data of this condition are summarized in Table III.

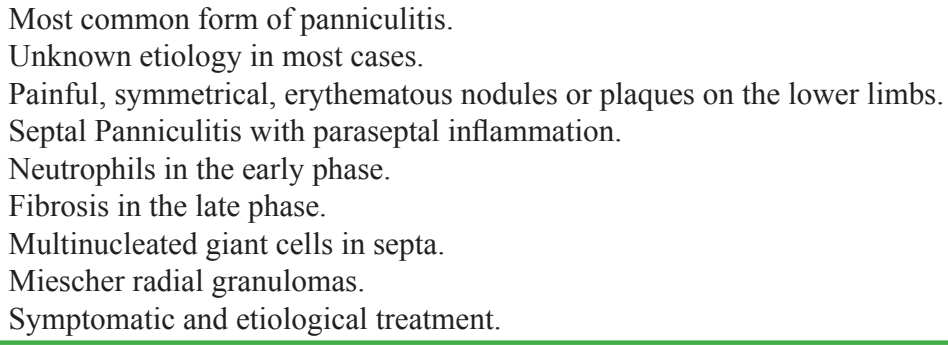

\section{Morphea Scleroderma Panniculitis}

Scleroderma is a disease of unknown etiology characterized by excessive deposition of certain components of the connective tissue and structural disorders of the blood vessels. Scleroderma includes two distinct processes: localized scleroderma or morphea and systemic scleroderma [11].

In localized forms, the process may extend into the subcutaneous tissue septum, from the deep dermis and sometimes the process is completely panniculitis, without involvement of the epidermis, dermis and skin appendages. These changes are particularly well developed in deep morphea [3].

Clinically it is characterized by indurated plaques or nodules, steady course or progressive growth, evolving to residual areas of atrophy and hyperpigmentation. The most frequently involved sites are the arms, shoulders and trunk [12]

The most characteristic histopathological finding of deep morphea is the presence of a marked fibrous thickening of the septum of the subcutaneous tissue and thickening of collagen fibers. Collagen replaces the fat normally present around the coils of the eccrine glands and beneath them, giving the impression that amounted eccrine glands within the dermis. The spaces between the collagen bands disappear, with accompanying atrophy, blood vessels and piloerector single muscle fibers remain. When the sclerotic process involves the thickness of the dermis and subcutis, it appears homogeneous eosinophilic. There mucinous changes in the interlobular septa, with particularly striking inflammation at the dermalsubcutaneous interface; lymphocytes surrounded by plasma cells predominate. There may be macrophages and eosinophils and, in some cases, considerable plasma cell numbers [3].

Topical, intralesional or systemic corticosteroids are the most used drugs. You can use alternatives such as penicillamine, topical tacrolimus, nifedipine more urea cream, oral pentoxifylline [3]. Main data of this condition are summarized in Table IV.

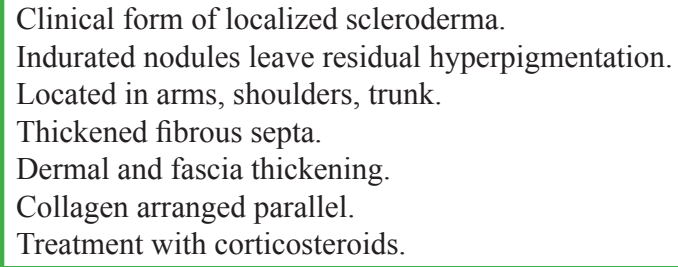




\section{Necrobiosis Lipoidica}

Necrobiosis lipoidica (NL) is defined as a process that causes skin collagen degeneration and palisaded granulomas, often associated with systemic diseases, particularly diabetes mellitus [13].

It was first described by Oppenheim in 1929 in a diabetic patient and called ,dermatitis atrophicans diabetic lipoidica”. In 1932 Urbach also recognized a second case in a diabetic patient and gave him the name "diabetic necrobiosis lipoidica" and suggested that a circulating toxin in these patients was responsible for the skin lesions.

In 1933 Balbi suggests that hyperlipidemia accompanying diabetes would be important in the pathogenesis of this process factor Goldsmith in 1935 reported the first case of necrobiosis lipoidica not associated with diabetes mellitus. Miescher and Leder in 1948 reported a series of non-diabetic patients who had lesions especially scalp, thinking of a new entity named „chronica et progressiva disciformis granulomatosis”. Winkelmann and Muller in 1966 established the association between necrobiosis lipoidica with diabetes mellitus in $0.3 \%$ of patients and $65 \%$ of cases of patients had abnormalities in glucose metabolism. Since then there have been many authors who have described cases in nondiabetic patients so it was decided to call this disease simply necrobiosis lipoidica (NL) [13].

The necrobiosis lipoidica is closely associated with diabetes mellitus, two thirds of these patients suffer and the remaining $1 / 3$ has an impaired glucose tolerance. Despite this, NL is rare and only $0.3 \%$ of diabetics suffer from it.

It affects more women and the female/male ratio is $3: 1$, the mean age of onset is between 30 and 45, appears at an earlier time when associated with diabetes and in nondiabetic late. Very few studies have been conducted in children. Is frequently observed in type 1 diabetes and in 7-30\% of type 2 diabetics, this gives a prevalence of $6.5 \%$ in type 1 diabetics and $0.4 \%$ in type 2 diabetes. The dermatosis can precede, coincide or occur after the diagnosis of diabetes [3]. In one study, preceded the onset of diabetes in $15 \%$ of patients, in $25 \%$ of patients appeared with the diagnosis of diabetes, and in $60 \%$ of patients appeared after diagnosis of diabetes. $66 \%$ of patients presenting with NL were diagnosed with diabetes mellitus and the rest showed abnormalities in glucose metabolism [13-15].

The cause of NL remains unknown. It has been reported in patients with various diseases. Many theories have been proposed to try to explain the pathogenic mechanisms of the NL and current theories postulate the following:

a. Microangiopathy. Appears to be the most important in pathogenesis. The vascular damage is preferentially localized in the basement membrane of small vessels, which is due to the combination of multiple factors. We found elevated levels of fibronectin, alpha-2- macroglobulin, ceruloplasmin in LN patients and granuloma annulare and diabetes mellitus and elevated haptoglobin, in patients without diabetes mellitus NL. These protein abnormalities could be responsible for angiopathy.

b. Inheritance. HLA types in 2 groups of diabetic patients with and without NL, in both groups a high frequency of HLA-DR4, B8 and CW3, and a low incidence of HLA -DR5 and DR7 were studied was observed. In agreement with previous reports of HLA patterns in diabetic patients, it is suggested that genetic factors do not play an important role in NL.

c. Immunological mechanisms. For some authors, the trigger factor for NL can be mediated by immune vasculitis due to evidence of immunoglobulin deposits $\operatorname{IgM}, \operatorname{IgA}$ and $\mathrm{C} 3$ in the walls of the affected vessels, plus IgM, C3 or fibrinogen in the dermo-epidermal junction in some patients. An increase of dendritic cells $\mathrm{S}-100$ positive has been detected in the epidermis $[3,17]$.

d. Alterations of collagen. It is accepted that the granulomatous response may be due to alterations of collagen. It is postulated that hyperglycemia causes increased activity of the aldolase -reducing enzyme, resulting in retention of water in the tissues, and destruction of collagen hyperhydration. Other studies reveal decreased concentration of hydroxyproline in the affected skin, with decreased collagen content and decreased number of fibroblasts. However, this hypothesis does not explain the cases where no hyperglycemia is detected $[3,17]$.

e. Sweat and neural abnormalities. The functioning of sweat glands is altered and the number of nerve endings in biopsies is found to be decreased. However, these changes appear to be the result of the local destruction of these structures by a primary inflammatory process $[3,17]$.

Clinically it is characterized as a chronic disfiguring process. Most lesions are located in the legs, especially in the pretibial region, although lesions on the face, scalp, arms, and trunk may also occur. The lesions are unique but may be multiple.

Initially presents as erythematous papules that increase in size and become plaques with a brownish yellow atrophic center with telangiectasias on the surface. The lesions may resolve spontaneously or become chronic with occasional ulceration. Painful ulcers occur in approximately $15 \%$ of cases. The occurrence of squamous cell carcinoma has been reported as a rare complication $[13,16,17,19]$.

Cases with atypical features are outlined, considering both these lesions are located outside of the legs (face, scalp, fingers, penis, nipples, postsurgical scars), as different morphology injuries that may occur as papules, nodules, morphoeic plaques, or ulcerated lesions and piercing injuries. It is clear from the published cases, these atypical forms are more frequently associated with systemic diseases, among which are inflammatory bowel disease (Crohn's disease, ulcerative colitis and intestinal bypass syndrome), sarcoidosis, rheumatic diseases, infectious, hematologic and ataxia-telangiectasia [17].

Histopathology found that the epidermis may be normal, ulcerated or atrophic. There are necrobiotic granulomas in the dermis. The necrobiotic pattern is more common in diabetics and those located in the legs [3].

The granulomatous process in NL extends from the deep dermis to the top of the septum of the subcutaneous tissue, resulting in a septal panniculitis. Necrobiosis in reaction areas of degeneration of collagen is observed. Collagen bundles may be thickened, chipped, or hyalinized amorphous anuclear and extend in different directions. Predominately in the lower third of the dermis and can reach the subcutaneous fat. Necrobiotic areas around lymphocytes, plasma cells, fibroblasts and epithelial cells are observed. In some areas, the inflammatory infiltrate is arranged in palisades surrounding areas of necrobiosis [3].

In the granulomatous reaction, collagen degeneration is moderate. Granulomas composed of histiocytes, epithelioid cells and multinucleated giant cells were observed. Around these granulomas there is no lymphocytic infiltrate. In older lesions, areas of hyalinization of collagen is observed. 
Yen Shan et al reported three cases with atypical histopathological features such as prominent tuberculoid granulomas, and the perineural inflammatory infiltrate predominantly lobular panniculitis [20].

Direct immunofluorescence of NL demonstrated in IgM, IgA and fibrinogen in the walls of venous vessels, causing vascular thinning. In nondiabetic vascular changes are not prominent.

\section{Treatment can be divided into 6 categories:}

1. Cutaneous blood flow enhancers: In this group the combination of high doses of aspirin with dipyridamole, stanozolol, ticlopidine, perilesional injections of heparin, pentoxifylline and prostagland E1 are cited all with varying results in different studies.

2. Corticosteroids: Topical and intralesional corticosteroids may reduce inflammation in active lesions or lesions on the edges of growth, but has little benefit in stable atrophic lesions, which could worsen atrophy thereof. Intralesional triamcinolone was reported as beneficial in active lesions. In two case reportsthe benefit of clobetasol propionate under occlusion was demonstrated and in two other studies the use of systemic corticosteroids in short pulses was suggested to be beneficial.

3. Surgery: NL tends to exhibit the phenomenon of Koebner so curative surgery can not be guaranteed.

4. Cutaneous healing enhancers: Various reports of efficacy of various topical preparations that act to promote cutaneous healing for ulcerated NL. One case report has described the efficacy of recombinant granulocyte macrophage colony stimulating factor topically to ulcerated NL in young patients with diabetes. Physical modifications of wound background is also beneficial in promoting wound repair. Promogran, a new protease modulating matrix, has been reported to be effective in wound healing. This preparation inactives metalloprotease and other enzymes found in large quantities to have a negative effect on wound healing. The bovine collagen gel applied in a nondiabetic patient under occlusion for 6 weeks, resulted in healing at 24 weeks with no recurrence after 5 months. In two diabetic women with ulcerated NL HBO achieved healing of the lesion after 98 and 113 sessions, this could be related to the correction of tissue hypoxia.

5. Immunomodulators: Within immunomodulatory efficacy in several studies of cyclosporine, mycophenolate mofetil, thalidomide, infliximab and tacrolimus are cited.

6. Miscellaneous: This group includes nicotinamide, clofazimine, chloroquine and topical tretinoin. A study of nicotinamide showed improvement in the lesions of NL in 8 of 15 patients. Clofazomine that has both anti-inflammatory and antimicrobial activity presented an effectiveness of $60 \%$. In two studies with topic tretinoin has reported an improvement of the atrophic component NL. Application NL benzoyl peroxide has been reported to ulcerated beneficial $[15,18]$. The main findings of this condition are shown in Table V.

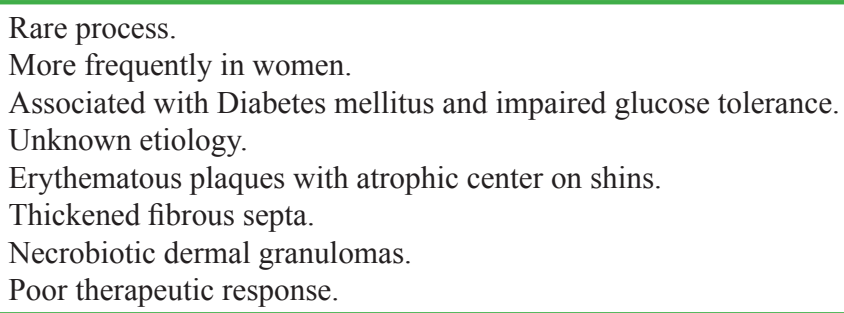

\section{Septal panniculitis with vasculitis \\ Polyarteritis Nodosa}

The term polyarteritis nodosa (PAN) includes systemic PAN, cutaneous PAN and microscopic PAN (microscopic polyangiitis) [23].

The limited variety of benign cutaneous polyarteritis nodosa was individualized by Lindberg in 1931 [23]

Both cutaneous and systemic PAN polyarteritis histologically characterized by necrotizing vasculitis of medium-sized arteries, but cutaneous PAN may be clinically differentiated from systemic polyarteritis because they involve organs. Systemic polyarteritis, by contrast, can affect any organ, especially the kidneys, heart and liver, and skin, in 50\% of cases. The largest series of cutaneous PAN not demonstrate development of systemic disease in a mean follow up of 7 years [21].

The etiology is so far unknown, although the demonstration of deposits of immune IgM and C3 in lesions of some vessels as well as the detection of circulating immune complexes in others, suggesting immune complex mediation. In some cases there is a high titer of antistreptolysin O (ASO).

Classic polyarteritis nodosa is a systemic necrotizing vasculitis of small and medium caliber arteries with poor prognosis and involvement of various organs, such as kidneys, liver, gastrointestinal tract and nervous system, skin manifestations may occur in $25 \%$ to $60 \%$ of cases [21].

On the other hand, cutaneous polyarteritis nodosa designates the exclusive skin involvement (deep dermis and panniculus), with a benignchronic course.

Skin lesions were distributed especially in the legs, but can occur in the upper limbs, face, scalp and mucous membranes [22].

Are erythematous and painful nodular lesions, the size will vary between 0.5 and $3 \mathrm{~cm}$, the number can vary, but reports from a few to over 100 .

It is commonly found in some patients, lesions and other recent developments, injuries later stages. Nodular lesions may evolve from a few weeks to over a year, with treatment evolve into violet color and may leave some hyperpigmented areas and in some cases leave scars. Nodules usually occur symmetrically, but in some cases the lesions may also be asymmetric. During exacerbations, some nodules can be reactivated in remission. Nodules usually occur singly or in groups $[21,22]$. 
According to the classification of Chenen 1989 and Daoud et al three types of skin lesions in PANC described in 1997: a mild form or class I characterized by nodular lesions and livedo reticularis, and between extracutaneous manifestations reported mild polyneuropathy.

Class II is characterized by addition of nodular lesions which may ulcerate and are quite painful, in this group of patients is also described polyneuropathy, fever, malaise and joint pain in the acute phase.

Class III has the characteristics of a PANC but skin lesions are characterized by livedo racemosa and necrotizing gangrene and also have a commitment to progressive musculoskeletal, mononeuritis multiplex, foot drop and some laboratory data autoimmune character. This class III is characteristic of a systemic PAN and is not observed in the cutaneous PAN [21]. The laboratory abnormalities are not too frequent in cutaneous
PAN. These deserve mention: elevated ESR, elevated streptococcal enzymes, leukocytosis, I antinucleolar antibody positive, positive cytoplasmic antibodies (ANCA) and elevated C-reactive protein [23].

The most effective treatment of cutaneous PAN is steroids, which are generally used to moderate doses (30-60 mg prednisone daily).

Other drugs used were colchicine ( 0.5 to $1 \mathrm{mg}$ orally daily) , azathioprine (50 mg daily, oral), methotrexate (10 mg weekly, orally, fractional ), dapsone (50 mg daily, po), cyclophosphamide $(1 \mathrm{mg} / \mathrm{kg} /$ day orally $)$. In cases involving streptococcal infections, penicillin and mycobacterial infection indicates clarithromycin ( $250 \mathrm{mg}$ twice daily, oral), ciprofloxacin at the same doses. 23 These findings are summarized in Table VI.
Localized form of polyarteritis nodosa.
Exclusive skin involvement.
Unknown etiology.
Painful erythematous nodules on the lower limbs.
Necrotizing vasculitis of small vessels in the dermis and subcutaneous.
Treatment with systemic corticosteroids.

Table VI. Summary of findings. Cutaneous polyarteritis nodosa.

\section{Surface Migratory Thrombophlebitis}

It is characterized by recurrent episodes of segmental thrombosis affecting the superficial veins of the lower extremities, trunk or abdomen.

It is characterized by the appearance of painful nodules distributed linearly along the limb and the evolution result in a palpable induration cord. Apart from the superficial veins of the lower limbs may be affected the lateral epigastric, thoracoepigastric or thoracic veins leading to visible or palpable cords in the chest wall. This variety is known as Mondor disease and has also been described in the axilla, groin and penis [24]. This form of septal panniculitis occurs in most patients without evidence of underlying disease. It is usually a complication of varicose veins. It can develop as a result of a primary or secondary hypercoagulable state. Primary hypercoagulable states, such as deficiencies of antithrombin III, heparin cofactor II, protein $\mathrm{C}$, protein $\mathrm{S}$ and factor XII. Fibrinolytic system disorders, dysfibrinogenemia and lupus anticoagulant are conditions that may be complicated by the development of this panniculitis. Also, it may be associated with underlying processes, such as venous varices, Behçet's syndrome, Burger's disease, drug abuse, infections, pregnancy or malignancies.
When the hypercoagulable state secondary to an underlying malignancy (Trousseau syndrome), the most common places where the primary malignancy sits are the pancreas, stomach, lung, prostate, colon, ovary and gallbladder [3].

Histology notes that recent injuries have an inflammatory infiltrate of polymorphonuclear predominance that affects the entire thickness of the wall of the vein and as the injury evolves polymorphonuclear are replaced by lymphocytes, histiocytes and occasional multinucleated giant cell. During evolution, the thrombus which occludes the lumen initially is replaced by recanalization and fibrosis. Thrombosis of these veins does not alter substantially the lobule oxygenation mode, so you do not find a significant accompanying panniculitis [24].

Treatment can be disappointing, with frequent recurrences. Measures such as relative rest and elastic compression stockings can improve the symptoms. In the case of Mondor, s disease lesions usually resolve in a few days, with a low recurrence rate. Most clinicians reserve the use of anticoagulants for patients with involvement of the deep venous system. In cases of treatment associated neoplasia addition thereof adjuvantly used anticoagulants, especially heparin [24]. A summary of findings are shown in Table VII.

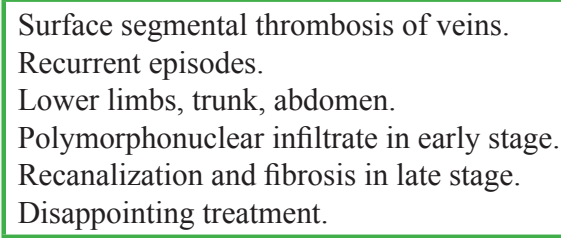

Table VII. Summary of findings. Surface migratory thrombophlebitis. 


\section{Study protocol of septal panniculitis}

- Clinical history, especially investigating the possible triggers and the existence of previous similar processes.

- Physical Examination.
- Analytical routine (complete blood count, biochemistry, coagulation).

- Excisional biopsy for histology and direct immunofluorescence. - Based on histological changes see Figure 1.

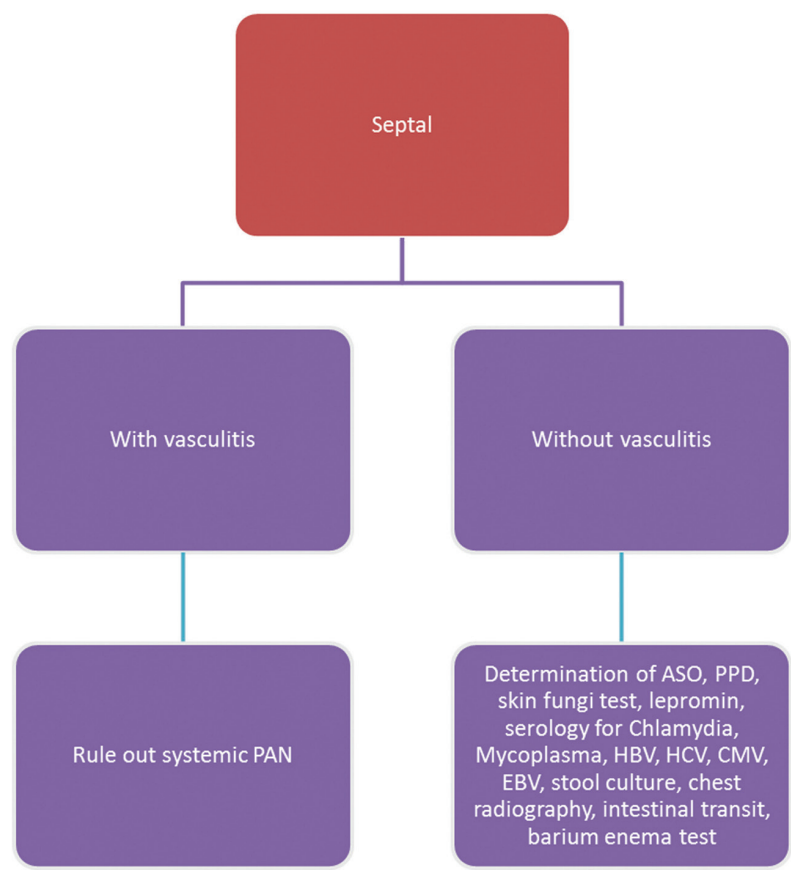

Figure 1. Study protocol of septal panniculitis based on histological findings.

\section{Presentation of a case of septal panniculitis}

Female, 67 years, diabetes treated with oral agents, chronic NSAIDs for osteoarthritis. Admitted to the emergency department with upper gastrointestinal bleeding gastric ulcers, drawing attention to physical examination leg ulcers 3 years of evolution. Several treatments used without improvement diagnosed as varicose ulcer.

Physical exam: ulcers, regular margins, net limits, fibrinous center, in lower $1 / 3$ of the lower limbs (Fig. 2a, b). Livedo reticularis in upper and lower limbs.
Histopathology: widening of subcutis septa by presence of abundant numbers of inflammatory cells, including macrophages, foamy predominate. Some neutrophils. Absence of vasculitis. Extension of the infiltrated to the dermis. Ziehl Neelsen for AFB + (fragmented bacilli) (Fig. 3a - c).

Auxiliary Studies: Analytical laboratory data normal except $\mathrm{Hb}$ $10.4 \mathrm{~g} / 1$. Positive cutaneous lymph for AFB.

Diagnosis: Reactional erythema nodosum.

Evolution: The patient died due to upper gastrointestinal bleeding.

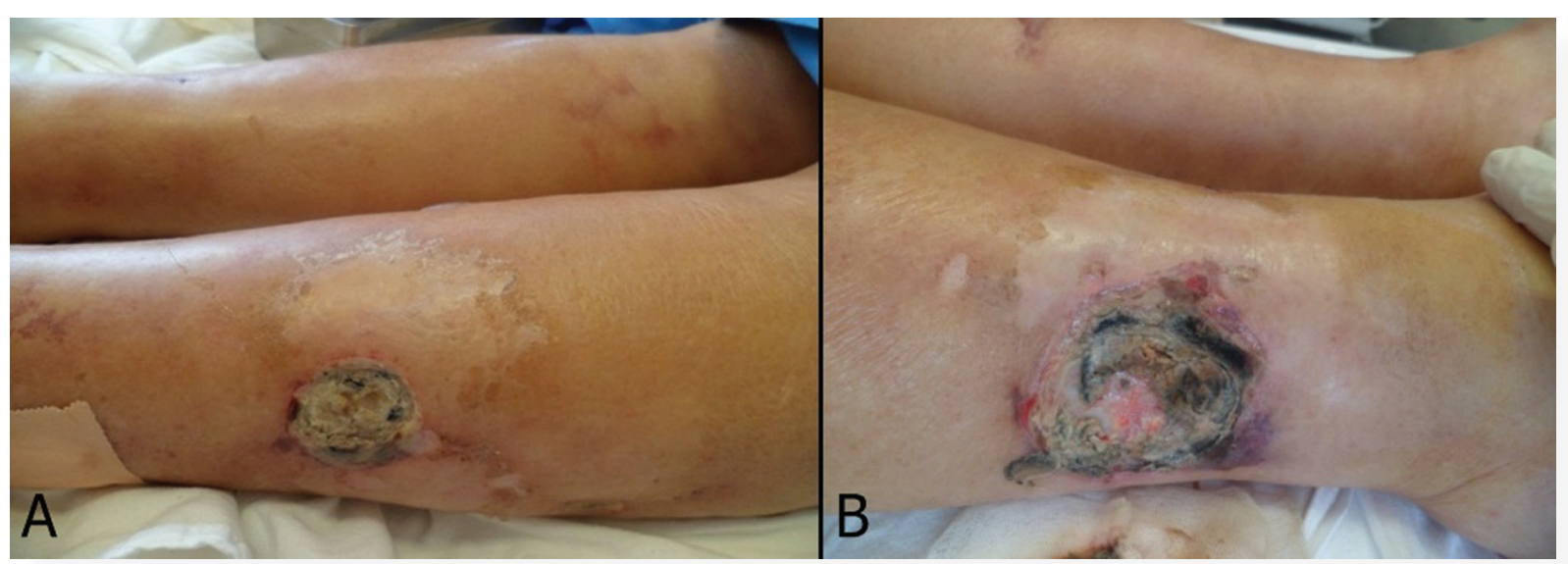

Figure 2A, B. Clinic. Ulcer with necrosis, fair edges, net limits, located in the anterior aspect of Both legs. 


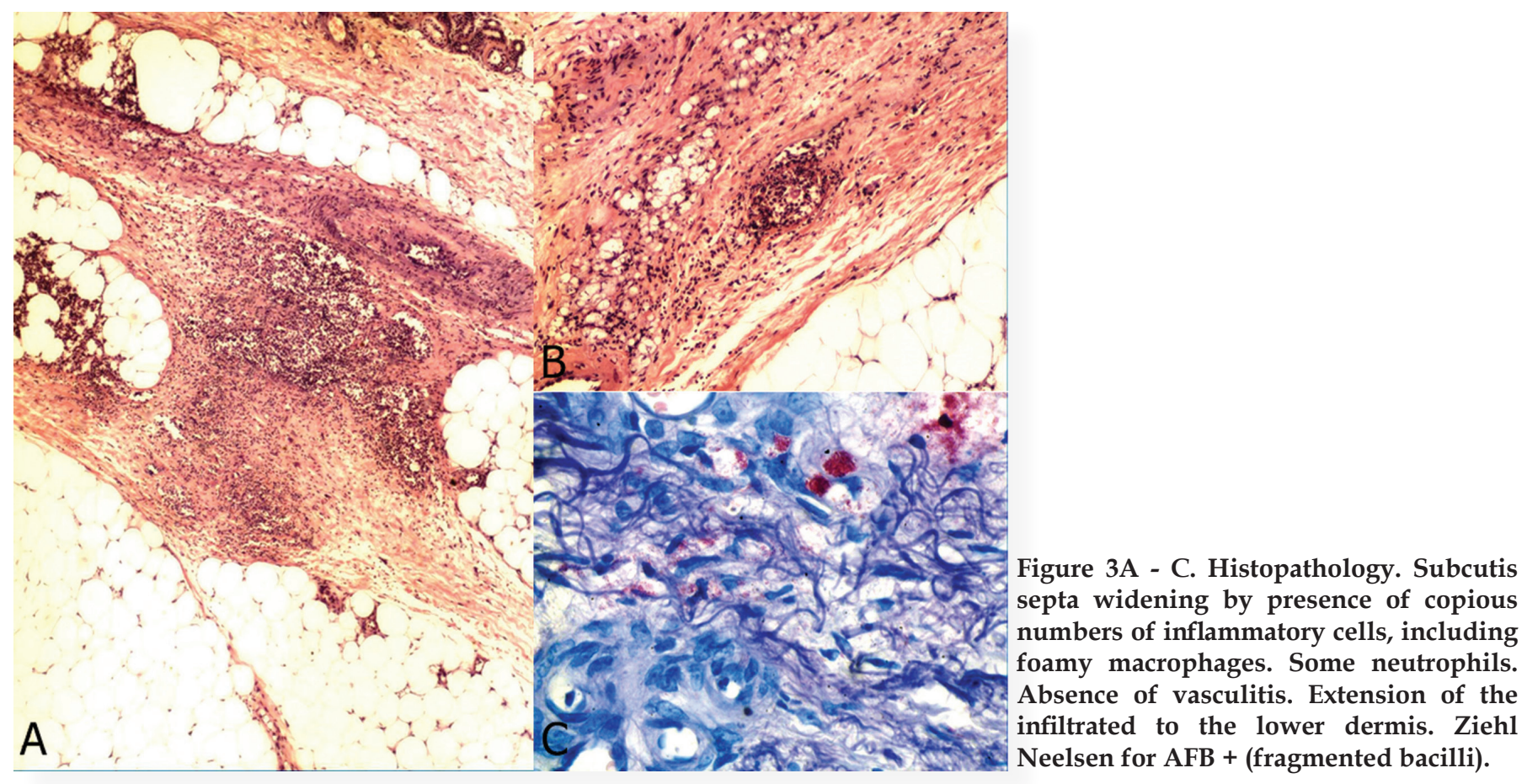

\section{REFERENCES}

1. Avilés Izquierdo JA, Ochaíta Lázaro P, Recarte Garcia-Andrade C, Izquierdo del Monte MG, Sánchez Granados A. JANUS. 2005; LXIX:42-4.

2. Vega Memije ME, Hojyo Tomoka MT, Soto LD, Sanchez L. Panniculitis. Peru Dermatol. 2006;16:36-61.

3. Sanchez I, Quesada A. Basic lesions in dermatology. Rev Med Costa Rica Centr. 2010;594:345-8.

4. Schwartz R. Erythema nodosum: a sign of Sistemic disease. Am Fam Physician. 2007;75:695-700.

5. Jareño A. Erythema nodosum. EAP Orcasitas. 2013;63:26-30.

6. González-Alvarez G, Cardona-Castro N. Erythema nodosum and its association with fungal infections. CES Rev Med. 2010;24:47-60. 7. Muñoz J, Garcia E, Berlango A, Calderon J, Montero F. Management of erythema nodosum in emergencies. Semergen. 2010;24:395-8.

8. Polcari I, Stein C. Panniculitis in childhood. Dermatol Ther. 2010;23:356-67.

9. Moreno A. Erythema nodosum. In: Herrera E, Moreno A. Dermatology: clinicopathologic correlation. 1 ed. Ed Menarini. Madrid. 2011.p:191-4.

10. Pastor JF. Linear scleroderma. Injury en coup de saber. An Esp Pediatr. 1998;49:499-502.

11. Macias L. Depressed indurated plaques on lower limbs. Peru Dermatol. 2004;14:211-4.

12. Santamaria V, Rodriguez E, Venadero F, Cortez B. Necrobiosis lipoidica. Presentaciónn of three cases and literature review. Easter Cent Rev Dermatol. 2001;10:154 -64.
13. Rodríguez-Acosta ED, Garcia-Hidalgo L, Saeb-Lima M, OrozcoTopete R. Prevalence of necrobiosis lipoidica in patients treated at the National Institute of Medical Sciences and Nutrition Salvador Zubirán. Rev Mex Dermatol. 2011;55:195-9.

14. Velasco AM. Necrobiosis lipoidica: update on its etiology and treatment. Intern Rev Dermocosm Dermatol. 2002;5:342-7.

115. Kota S, Jammula S, Meher L. Necrobiosis lipoidica diabeticorum. A case based review of the literature. End Ind Met J. 2012;16:614-20. 16. Segurado Rodríguez MA, Garcia Donoso C, Gamo Villegas R, Guerra Tapia A, Iglesias Diez L. Nodular necrobiosis lipoidica: report of a case. Dermosifiliogr Proceedings. 2002;93:555 -7.

17. Tidman M, Duncan C. The treatment of necrobiosis lipoidica. $\mathrm{Br}$ J Dis Vasc Diabetes. 2005;5:37-41.

18. Gudi V, Campbell S, Gould D, Marshall R. Squamous cell carcinoma in an area of necrobiosis lipoidica diabeticorum: a case report. Clin Exp Derm. 2000;25:597-9.

19. Yen P, Wang K, Chen W, Kang Y. The many faces of necrobiosis lipoidica: a report of three cases with histologic variations. Dermatologica Sinica. 2011;29:67-71.

20. Quintana G, Rodriguez G. Polyarteritis nodosa atypical cutaneous. Rev Colomb Reumatol. 2008;20:320-5.

21. Cardenas L, Parsons A, Sangueza O. Cutaneous polyarteritis nodosa. Rev. Asoc Colomb Dermatol. 2010;18:40-2.

22. Viglioglia P. Polyarteritis nodosa. Act Dermatol Ther. 2008;31:238-42.

23. Laguna C. Surface migratory thrombophlebitis. Skin. 2011;26:391-4.

24. Laguna C, Alegrea V, Perez A. Migratory superficial thrombophlebitis: clinical and histological review of 8 cases. Proceedings Dermosifiliogr. 2008;99:390. 